Citation: L. Maffione (2020) Didattica Inclusiva a Distanza: tecnologie e tecniche per l'Inclusione di studenti con disabilità visiva. Media Education 11(2): 179-186. doi: 10.36253/me-9608

Received: August, 2020

Accepted: October, 2020

Published: December, 2020

Copyright: ( $) 2020$ L. Maffione. This is an open access, peer-reviewed article published by Firenze University Press (http://www.fupress.com/me) and distributed under the terms of the Creative Commons Attribution License, which permits unrestricted use, distribution, and reproduction in any medium, provided the original author and source are credited.

Data Availability Statement: All relevant data are within the paper and its Supporting Information files.

Competing Interests: The Author(s) declare(s) no conflict of interest.
Best Practices

\section{Didattica Inclusiva a Distanza: tecnologie e tecniche per l'Inclusione di studenti con disabilità visiva}

\author{
Online Inclusive Education: technologies and didactic \\ techniques for inclusion of students with visual disabilities
}

\section{LUCiA MAFFione}

MI, Ministero Istruzione

E-mail: prof.maffione@gmail.com

\begin{abstract}
This contribution illustrates the activation of an inclusive teaching practice realized during the suspension of face-to-face lessons, due to the Covid 19 epidemic. The purposes of the intervention were: to respond to the student's learning special needs, with visual disabilities and attending the Secondary School, as well as to achieve the objectives of her individualized educational plan. Throughout the article many various didactic techniques, software, aids, e-tivity and online open resources are presented to implement inclusive distance learning.
\end{abstract}

Keyword: inclusion, teaching technologies, technical aid, online teaching resources.

Riassunto. Il presente contributo illustra l'attivazione di una didattica inclusiva attuata nell'anno scolastico 2019/2020 a seguito della sospensione delle lezioni in presenza, a causa dell'epidemia da Covid 19. La finalità dell'intervento è stata quella di rispondere ai bisogni educativi speciali di una studentessa ipovedente, frequentante la Scuola Secondaria di II grado. Per realizzare una didattica a distanza accessibile ed inclusiva sono stati utilizzati: vari software, ausili, risorse in rete e specifiche tecniche e strategie, risultate molto utili per il raggiungimento degli obiettivi del PEI.

Parole chiave: ausili, inclusione, tecnologie didattiche, risorse didattiche in rete.
Contesto di riferimento: Scuola Secondaria di II grado, Barletta

Destinatario: Studentessa di anni 15 ipovedente

Durata: Le tecnologie didattiche illustrate nel seguente contributo sono state utilizzate durante il periodo di sospensione delle attività in presenza, a seguito dell'emergenza da Covis-19. Nello specifico nel periodo compreso tra marzo 2020 e giugno 2020 .

Tecnologie utilizzate: Pc e smartphone, siti internet, piattaforma "Edmodo", piattaforma "Padlet", "Google Documenti", "Google Drive", "Prezi”, softwa- 
re "Balabolka", sintesi vocale, piattaforma "Youtube", "WhatsApp".

Aree di competenza mediale: Lettore, fruitore.

\section{QUADRO TEORICO DI RIFERIMENTO}

Per 'didattica inclusiva' s'intende quell'insieme di azioni quali: "progettazione, realizzazione e valutazione di pratiche educative che attivano i processi di apprendimento e partecipazione di tutti gli alunni e di tutte le alunne» (Heidrun, 2019, p.109) all'interno di contesti educativi, nel pieno rispetto delle differenze individuali di ciascun apprendente.

Proprio perché include tutti i discenti, i destinatari della didattica inclusiva non sono solo i cosiddetti BES ${ }^{1}$, studenti con bisogni educativi speciali, bensì ogni studente del gruppo classe, accolto e valorizzato dal 'docente inclusivo', alla luce delle sue specificità individuali quali: fattori cognitivi, psicologici, affettivi, caratteriali etc. Non a caso, la finalità ultima della didattica inclusiva è proprio quella di mettere tutti gli studenti nelle condizioni di raggiungere il successo formativo, rimuovendo ogni potenziale barriera che possa ostacolarlo.

Il modello teorico a cui è possibile legare concettualmente e metodologicamente la didattica inclusiva è rappresentato dall'Universal Design for learning (Booth \& Ainscow 2002). Questo modello fornisce molteplici spunti e materiali per realizzare ambienti di apprendimento di autentica inclusività. Due concetti chiave proposti dall'Universal Design for learning sono:

- la metodologia 'uguale per tutti' risulta ormai obsoleta ed inapplicabile al coevo contesto contemporaneo, poiché come la psicologia ci ha insegnato, ogni individuo - con o senza disabilità - apprende in modo diverso.

- risulta fondamentale adottare una didattica multimodale: differenziata cioè nei mediatori utilizzati, nei materiali e nella proposta di diversificati contenuti da approfondire, allo scopo di rispondere in maniera personalizzata alle specificità cognitive e/o motivazionali degli apprendenti.

\section{MOTIVAZIONE}

L'emergenza Covid ha posto ogni insegnante dinanzi ad un'inedita sfida educativa: garantire il diritto allo studio di tutti gli studenti attuando un'efficace didattica a distanza.

Se nel 'periodo pre-Covid' valutazioni e/o riflessioni sull'E-Pedagogy (Elliot, 2008) o sulla "Pedagogia 2.0" (McLoughlin \& Lee 2011) coinvolgevano esclusivamente 'addetti ai lavori', ricercatori e studiosi di media education, l'obbligatoria attivazione della didattica a distanza ha messo l'e-learning al centro della riflessione insegnativa di ciascun docente, di ogni ordine e grado. Variabili quali: disponibilità di ausili e connessioni tra gli studenti, gestione del carico di lavoro, sostegno alla motivazione, overload informazionale, suddivisione di tempi e scadenze, progettazione di modalità di verifica specifiche, e personalizzazione di materiali e delle e-tivity per assicurare l'inclusione di studenti con bisogni educativi speciali, sono diventate oggetto di interrogativi da parte di ogni formatore, trasformatosi all'improvviso in un formatore a distanza.

Le suddette valutazioni, inoltre, sono risultate ancora più cruciali per i docenti di sostegno, i quali hanno continuato a svolgere il delicato ruolo di 'ponte' tra docenti curriculari ed esigenze specifiche degli studenti di riferimento, pur rimanendo anch'essi fisicamente a distanza dagli apprendenti.

L'inaspettato cambiamento ha chiesto a ciascun insegnante specializzato di ripensare la didattica inclusiva per declinarla nella nuova 'modalità' a distanza. In particolare, il docente di sostegno ha dovuto "hacerse presentes" (Rappoport et al. 2020 p.4) nella pratica didattica con gli studenti di riferimento in diversi modi:

- Scegliendo i giusti strumenti informatici e le modalità comunicative appropriate in base alle specifiche necessità del discente;

- Rendendo accessibile i contenuti attraverso attività di personalizzazione o individualizzazione dei materiali di studio;

- Differenziando e/o semplificando le modalità di lavoro delle attività, in base alle finalità didattiche ed educative da perseguire;

- Assicurando un feedback costante ed una guida 'da remoto’ per momenti di difficoltà e/o stress psicologico.

\section{DESTINATARIO DELL'INTERVENTO}

La destinataria del seguente intervento didattico è stata una studentessa di quindici anni, Angela (nome di fantasia), frequentante la Scuola Secondaria di II grado, con una programmazione paritaria ed affetta da una disabilità sensoriale. Nello specifico, la studentessa è ipovedente: riesce a leggere testi scritti solo con un adeguato ingrandimento del carattere; discrimina immagini e lettere proiettate su un monitor (di LIM o pc) solo da una distanza molto ravvicinata e con un adeguato ingrandimento. A volte Angela tende a sottostimare il proprio deficit sensoriale, sottoponendosi ad un eccessivo stress visivo ed emotivo. La videoscrittura con lente di ingrandimento, l'uso di ausili informatici 
quali: audiolibri e sintesi vocale rappresentano tecnologie didattiche fondamentali per l'inclusione didattica e la compensazione del deficit sensoriale.

\subsection{Le specificità della Disabilità visiva: riflessioni peda- gogiche}

Per disabilità visiva s'intende la perdita totale o parziale della funzione visiva e, tale disabilità si colloca all'interno della macro area dei deficit sensoriali.

La compromissione, più o meno grave, del senso della vista necessita di una riflessione pedagogica speciale, oltreché opportune scelte metodologiche e didattiche sin dalla fase di progettazione di ciascun intervento didattico.

Tra le necessarie azioni da realizzare ricordiamo:

- Assicurare un contesto accessibile e privo di barriere architettoniche, e favorire la conoscenza e familiarizzazione degli ambienti al soggetto ipovedente, affinché possa mantenere un relativo livello di autonomia, in base alla gravità del deficit sensoriale.

- Sostituire i mediatori iconici con mediatori di tipo uditivo, prediligendo spiegazioni orali a proiezioni di immagini o video, o comunque assicurare un commento descrittivo di quanto proiettato alla classe, per realizzare un intervento di insegnamento/ apprendimento autenticamente inclusivo.

- Educare il discente all'uso di tecnologie ed ausili fondamentali quali: sintesi vocale, riconoscimento vocale, e strumenti di accessibilità presenti in ogni dispositivo tecnologico, per favorire l'autonomia dello studente. Non a caso l'approccio bio-psico-sociale proprio dell'ICF, riconosce un ruolo importante agli ausili didattici, in quanto essi rappresentano fattori contestuali in grado di facilitare il raggiungimento degli obiettivi prefissati e, per questo, potenti facilitatori dell'apprendimento.

\section{SOSTEGNO A DISTANZA: DALLA DIFFICOLTÀ NASCE L'OPPORTUNITÀ}

L'articolo 2 del "Manifesto per la Repubblica Digitale"2 è dedicato alla Cittadinanza digitale, ed afferma:

Il digitale progettato ponendo attenzione ai diritti dei cittadini può diventare la lingua comune nel dialogo tra cit-

\footnotetext{
${ }^{2}$ La Repubblica digitale è un'alleanza tra soggetti pubblici e privati, coordinata da un comitato interministeriale e guidato dal Ministro per l'innovazione tecnologica e la digitalizzazione. Nel quadro della strategia "Italia 2025", tra gli obiettivi posti dalla Repubblica Digitale abbiamo: riduzione del fenomeno dell'analfabetismo digitale, incremento della percentuale di specialisti ICT esperti nelle tecnologie emergenti.
}

tadini, amministrazioni pubbliche e imprese e contribuire al superamento delle diseguaglianze. Pubblico e privato rendono disponibili i propri servizi in forma digitale in maniera accessibile e a misura di cittadino senza creare nuove barriere tecnologiche e abbattendo quelle esistenti ${ }^{3}$.

Nonostante i contemporanei nativi digitali siano sempre iperconnessi ed ormai identificabili con la cosiddetta 'Generazione app' (Gardner\& Davis 2014), spesso 'assuefatti' all'uso di applicazioni più o meno utili, ad uno sguardo più attento, è possibile notare come le loro competenze digitali nell'uso di tecnologie utili per il miglioramento delle prestazioni scolastiche e/o professionali risultino ancora poco sviluppate. La cosiddetta 'alfabetizzazione digitale', che comprende anche abilità basilari come: gestire un account di posta elettronica e delle sue principali funzionalità, realizzare il backup on line, convertire in diversi formati i file, o conoscere i principali menu di formattazione testuale, non sono ancora dominio di tutti i nativi digitali.

Il ricorso 'coatto' alla didattica a distanza ha messo in luce le suddette carenze di alfabetizzazione tra gli apprendenti, ed ha inoltre confermato come la questione del divario digitale, sia in termini di dotazione strumentale che in termini di competenza d'uso, sia tutt'altro che superata.

Di conseguenza, avvicinare studenti con BES alla $\mathrm{DaD}$ non è sempre facile: oltre alla mancanza di competenze informatiche, nel caso di studenti con bisogni educativi speciali, si aggiungono le "barriere digitali" legate alla fruizione dei contenuti, soprattutto per studenti con DSA (in primis i dislessici) o per i disabili sensoriali. Una grande risorsa, in questi casi, è rappresentata dalla multimodal pedagogy (Bezemer \& Kress, 2016), realizzabile anche attraverso la didattica digitale, che permette ai docenti di utilizzare canali sensoriali alternativi, di compensare deficit e disturbi specifici ed includere ciascun apprendente, anche attraverso software, ausili e risorse in rete.

D'altro canto, l'attivazione della $\mathrm{DaD}$ ha determinato una maggiore frequenza d'uso delle tecnologie didattiche digitali che, grazie alla guida dei docenti, (trasformati nel frattempo anche in 'tutor informatici') hanno sviluppato o migliorato significativamente la competenza digitale degli studenti.

In particolare, l'esperienza della $\mathrm{DaD}$ con la studentessa Angela le ha consentito di:

- usare quotidianamente le varie funzioni presenti in G-mail;

- comprendere l'utilità del sistema di archiviazione di Google Drive;

\footnotetext{
${ }^{3} \mathrm{https} / / /$ repubblicadigitale.innovazione.gov.it/it/il-manifesto/
} 
- utilizzare gli strumenti di correzione e di accessibilità offerti da Google Documenti;

- conoscere ed usare autonomamente strumenti per video conferenze quali Hangout o Meet;

- Familiarizzare con l'interfaccia e le funzionalità della piattaforma Edmodo.

Inoltre, per favorire l'autonomia della studentessa Angela nel discriminare lettere e simboli con dimensioni ridotte, sono stati utilizzati i seguenti ausili e software per compensare il suo deficit visivo:

- Lente di ingrandimento del $\mathrm{pc}^{4}$

- App di "Trascrizione vocale istantanea", scaricabile ed utilizzabile con smartphone ${ }^{5}$

- "Teamviewer", ovvero un software proprietario per controllo remoto, condivisione desktop, riunioni online, conferenze Web e trasferimento di file tra computer.

In particolare, il programma "Teamviewer"6 è stato utilizzato dal docente specializzato per controllare da remoto il pc della discente nei seguenti casi:

- Per aiutare la studentessa ad individuare file o comandi nel pc;

- Per correggere in tempo reale quanto realizzato su file dalla studentessa;

- Per individuare errori procedurali durante lo svolgimento di attività in sincrono;

- Per svolgere attività in modo collaborativo;

- Per risolvere problemi tecnici legati al dispositivo informatico.

Naturalmente, l'iniziale senso di smarrimento è stato inevitabile, ritrovarsi all'improvviso a scrivere ed inviare compiti e verifiche online, conoscere, ambientarsi e 'dialogare' all'interno delle piattaforme digitali ha richiesto un breve periodo di 'rodaggio' per la studentessa, superato il quale è stato possibile lavorare con serenità e profitto.

4.1 Tecnologie didattiche per gli obiettivi del Piano Educativo Individualizzato

In coerenza con la Diagnosi Funzionale ed il Profilo di Funzionamento Funzionale, il P.E.I elaborato per l'anno scolastico 2019/2020 a favore della studentessa ha previsto il raggiungimento dei seguenti obiettivi:

1. uso del computer, di tastiere speciali, dei comunicatori, del cellulare per lo studio scolastico e domesti-

\footnotetext{
${ }^{4}$ Attivabile su tutti i pc tramite il menu: Impostazioni Pc/ Accessibilità/ Lente di ingrandimento

${ }^{5}$ Un guida sull'uso è disponibile online: https://www.youtube.com/ watch?v=65YNG20zxiI

${ }^{6}$ Scaricabile al sito: https://www.teamviewer.com/it/download/windows/
}

co (dominio d3601dell'ICF).

2. il potenziamento dell'espressione scritta (dominio d1702 dell' $\mathrm{ICF}^{7}$ );

Il perseguimento dei suddetti obiettivi è stato possibile sfruttando a pieno le potenzialità di strumenti informatici e di software didattici specifici, i quali grazie ad un'iniziale alfabetizzazione ed un uso costante nella pratica didattica, hanno permesso di compensare il deficit visivo della discente.

\subsection{Promuovere l'uso di ausili e tecnologie digitali attraver- so la $\mathrm{DaD}$}

Per il raggiungimento del primo obiettivo previsto dal PEI della studentessa, ovvero incrementare l'uso del computer, di tastiere speciali, dei comunicatori, del cellulare per lo studio scolastico e domestico (dominio d3601dell'ICF), sono state adoperate tecnologie ed ausili di diverso tipo e con specifiche finalità.

Nella fattispecie, per massimizzare l'accessibilità visiva dei contenuti da fruire, sono state gradualmente introdotte le seguenti tecnologie:

- Sintesi vocale

- "Padlet"

- Presentazioni con "Prezi"

- Audiolibri

Nel dettaglio, la sintesi vocale è stata utilizzata sia per la lettura di documenti scritti che per la conversione in audio di materiali di testo da studiare. Il materiale testuale è stato convertito in materiale in formato audio mp3 per la facilità di fruizione anche da dispositivi mobili.

Tutti i file audio realizzati sono stati poi raccolti e pubblicati su una lavagna online, "Padlet", con delle pagine specifiche create per ciascuna materia. L'indirizzo della lavagna online creata su "Padlet" è stato poi condiviso con la studentessa. I vantaggi offerti dalla piattaforma "Padlet" sono stati molteplici:

- Uso intuitivo ed immediato;

- Accessibilità da ogni dispositivo;

- Possibilità di caricare diversi formati: audio, video, link, testo, immagini, audio (in coerenza con la pedagogia multimodale);

\footnotetext{
${ }^{7}$ ICF è l'acronimo inglese di "International Classification of Functioning, Disabilities and Health", ovvero "Classificazione Internazionale del Funzionamento della Disabilità e della Salute" ed è il documento elaborato dall'Organizzazione Mondiale della Sanità per descrivere e misurare la salute e le disabilità della popolazione (Ianes, 2019). Una versione online è disponibile su: https://www.reteclassificazioni.it/portal_main. php?portal_view=public_custom_page\&id=85
} 
- Possibilità di fruizione dei contenuti in tempi e modi personalizzabili;

- Uso collaborativo della pagina attraverso la condivisione dell'indirizzo internet.

Il quotidiano coinvolgimento della studentessa nella didattica a distanza le ha, inoltre, consentito di dedicare più tempo all'"esplorazione" di risorse di studio presenti in rete e, in particolare, le ha dato la possibilità di conoscere due strumenti molto utili ed inclusivi per lo studio: le presentazioni di "Prezi" ed i siti internet per scaricare audiolibri.

Il sito "Prezi" permette non solo di realizzare, ma anche di consultare mappe ipermediali su moltissimi argomenti di ogni disciplina, e offre una prospettiva approfondita sui vari temi. Ad esempio, per ripassare argomenti di storia dell'arte è stata realizzata una mappa ipermediale contenente immagini e spiegazioni audio delle diverse opere d'arte studiate ${ }^{8}$.

Particolarmente importante è stata la conoscenza di specifici siti internet che danno accesso gratuito a numerosi audiolibri, preziosi ausili per compensare le disabilità visive, appartenenti alla letteratura classica e contemporanea. Nello specifico, è stato fornito un video tutorial, disponibile su "YouTube", su come operare in ciascuna piattaforma con l'indicazione dei principali indirizzi on line in cui reperire audiolibri in modo sicuro e legale .

\subsection{Tecnologie didattiche per potenziare l'espressione scritta in $\mathrm{DaD}$}

Per quel che riguarda il secondo obiettivo previsto dal PEI della studentessa, ovvero il potenziamento dell'espressione scritta in lingua madre, si è gradualmente spiegato e incoraggiato l'uso delle funzionalità presenti tra gli Strumenti di "Google Documenti", ovvero il controllo ortografico e grammaticale, oltreché il dizionario e la possibilità di scrivere collaborativamente con compagni e con il docente specializzato attraverso la funzione Condividi. Da non trascurare inoltre altri strumenti utili ai fini dell'inclusione didattica quali: la digitazione vocale, l'attivazione della lente di ingrandimento ed il supporto per lo screen reader, presenti sempre nel menu Strumenti di "Google Documenti".

Oltre ad utilizzare i suddetti importanti ausili compensativi, è stata svolta una lezione in Dad sui comple-

8 https://prezi.com/eafcwlgwqrya/copy-of-copy-of-learn-prezifast/?present $=1$

${ }^{9}$ I link suggeriti sono stati: https://www.youtube.com/watch?v=1MHevlXBfQ e https://www.raiplayradio.it/programmi/adaltavoce/archivio/ audiolibri/ menti indiretti. Il seguente nucleo tematico è stato estrapolato dalla programmazione di lingua italiana svolta dall'intera classe, poiché come già detto, la studentessa segue una programmazione comune al gruppo classe.

Gli obiettivi previsti dall'attività sono stati:

(Conoscenze)

- Conoscenza delle funzioni dei principali complementi indiretti;

- Conoscenza della struttura linguistica alla base di ciascun complemento indiretto.

(Abilità)

- Utilizzare l'attenzione focalizzata e l'attenzione selettiva per individuare informazioni specifiche all'interno di video, tabelle e mappe mentali;

- Potenziare la memoria uditiva attraverso l'ascolto di brevi mp3;

- Utilizzare risorse on line per migliorare la comprensione e l'apprendimento;

- Produrre frasi corrette con i complementi.

La Competenza chiave coinvolta è stata quella Alfabetico-Funzionale, ed i settori disciplinari implicati sono stati: Lingua, letteratura e cultura italiana e Tecnologia Informatica.

Si è scelto di proporre la seguente attività in modalità asincrona allo scopo di rispettare i tempi di apprendimento della studentessa, ed evitare di creare stress emotivo o ansia a causa di scadenze temporali stringenti.

Per quel che riguarda l'articolazione dell'attività, si è proposto una breve unità didattica con una struttura 'balboniana' (Balboni, 2002, pp.100-101), composta dalle seguenti cinque fasi:

1. Lead-in: per introdurre la tematica e stimolare la curiosità dello studente, sono stati forniti alcuni advance organizer, attraverso la visione di un video introduttivo sull'argomento 'Complementi Indiretti' estrapolato dalla piattaforma "YouTube";

2. Globalità: per presentare in modo globale la tematica e fornire una visione d'insieme dell'argomento, si è chiesto alla studentessa di analizzare una mappa mentale contenente tutti i complementi in forma sintetica, disponibile online al sito https://www.mappe-scuola.com/, nella sezione dedicata alla grammatica italiana;

3. Analisi: nella fase di preparazione, il docente ha reso accessibile l'input per la discente, convertendo le spiegazioni dei complementi in formato audio mp3 con il software "Balabolka"10. Successivamente il

\footnotetext{
${ }^{10} \mathrm{Il}$ software "Balabolka" permette di leggere testi scritti con la sintesi vocale e di salvarli e convertirli in vari formati, tra cui il formato mp3. Il programma è scaricabile gratuitamente al seguente indirizzo: https://
} 
materiale esplicativo in formato audio è stato raccolto e pubblicato on line sulla piattaforma "Padlet"11. In questa fase è stato importante comprendere ed analizzare nel dettaglio gli input. Allo studente si è chiesto di ascoltare con attenzione le spiegazioni e gli esempi forniti.

4. Sintesi: attraverso il ricorso al mediatore visivo della tabella, si sono presentati gli elementi logici presentati in modo riassuntivo e schematico.

5. Riflessione: alla studentessa è stato chiesto di registrare un audio in cui fornire brevi frasi contenenti i complementi studiati. Si è scelto una prova di produzione orale, per minimizzare lo sforzo visivo e, al contempo, focalizzare l'attenzione della discente sul contenuto sintattico della produzione. Questa prova è stata considerata per la valutazione, ed i criteri di riferimento utilizzati per la valutazione sono stati:

- Correttezza sintattica,

- Competenza lessicale,

- Coerenza semantica.

La durata complessiva dell'attività è stata di 2 ore.

In ciascuna delle suddette fasi si è fatto uso di specifiche tecnologie e/o risorse digitali in base all'obiettivi didattico da perseguire, oltreché al bisogno specifico dell'apprendente.

Non si sono verificati momenti di difficoltà nella realizzazione dell'attività. Tuttavia sono stati

necessari dei tempi più lunghi rispetto a quelli previsti nella progettazione per consentire all'alunna un buon approfondimento dell'argomento.

Gli obiettivi previsti sono stati raggiunti, poiché la studentessa ha mostrato molto interesse nei confronti delle attività proposte, e il prodotto d'apprendimento finale è risultato corretto dal punto di vista sintattico e semantico. Le attività che hanno avuto maggior successo e hanno interessato la studentessa sono state: il video introduttivo e la mappa mentale proposta sulla tematica oggetto della lezione.

4.4 Strategie e tecniche per la gestione dello stress emotivo in Dad

Nei momenti di maggiore tensione emotiva e preoccupazione da parte della studentessa, manifestatisi soprattutto in prossimità di verifiche online o dinanzi a compiti nuovi o percepiti come difficili, si è cercato di adottare una comunicazione positiva, attraverso bre-

\footnotetext{
balabolka.it.softonic.com/

${ }^{11}$ Il padlet creato è disponibile al seguente indirizzo: https://it.padlet. com/prof_maffione/6iabcxn9j7y8zeru
}

vi messaggi audio inviati con l'app "WhatsApp" o con chiamate e/o videochiamate nei momenti di bisogno.

La suddetta comunicazione positiva è stata realizzata attraverso le seguenti tecniche:

- Uso di un'impostazione positiva del discorso;

- Praticare l'ascolto attivo, attraverso cui si «invia la propria decodifica del messaggio del mittente» (Gordon, 1970, p.39) e quindi riformulando i messaggi prodotti dalla studentessa;

- Evidenziare i punti di forza ed i progressi già compiuti dell'allieva;

- Evitare di incentrare la comunicazione su tutto ciò che la studentessa non ha fatto (mancate consegne, omissioni, etc.);

- Evitare il rimprovero per scongiurare fenomeni di chiusura o aggressività.

Altre strategie utilizzate sono state: la flessibilità dei tempi di consegna dei compiti on line, allo scopo di permettere alla studentessa di lavorare secondo propri tempi e ritmi di apprendimento. Come pure l'utilizzo di un feedback costruttivo: per ciascuna correzione è stata premura del docente utilizzare un feedback partendo dai punti di forza presenti. Come ad esempio:

- Sottolineare tutto quello che è stato fatto in maniera corretta nel compito;

- Evidenziare il livello di competenza raggiunto (docente: "adesso hai imparato a fare...");

- Definire il successivo step dell'apprendimento (docente: "ti rimane da imparare questo...") in coerenza con la Zona di Sviluppo Prossimale di Vygotskij.

\section{CONCLUSIONI}

Per verificare quali delle tecnologie didattiche utilizzate fossero risultate più efficaci per la studentessa di riferimento, le è stato chiesto di fornire un feedback, rispondendo ad un breve questionario. Dal sondaggio ${ }^{12}$ è emerso che le tecnologie e le risorse apprezzate maggiormente dalla studentessa sono state: "Youtube", Software "Trascrizione istantanea", "Padlet", la sintesi vocale, e "TeamViewer".

Il periodo di sospensione delle attività didattiche in presenza dell'anno scolastico 2019/2020, a causa dell'emergenza sanitaria da CoVID-19, ha rappresentato per Angela, come per tutti i discenti, senz'altro una barriera al raggiungimento del successo scolastico, ma le tecnolo-

\footnotetext{
${ }^{12}$ Il questionario completo e le relative risposte raccolte sono consultabili al seguente link: https://docs.google.com/forms/d/10JiQFBR7Ng2_ W7DtnmtlvsUy37PyCTzu5A-9V3xfuco/edit\#responses
} 
Tabella 1. Strutturazione dell'intervento didattico a distanza.

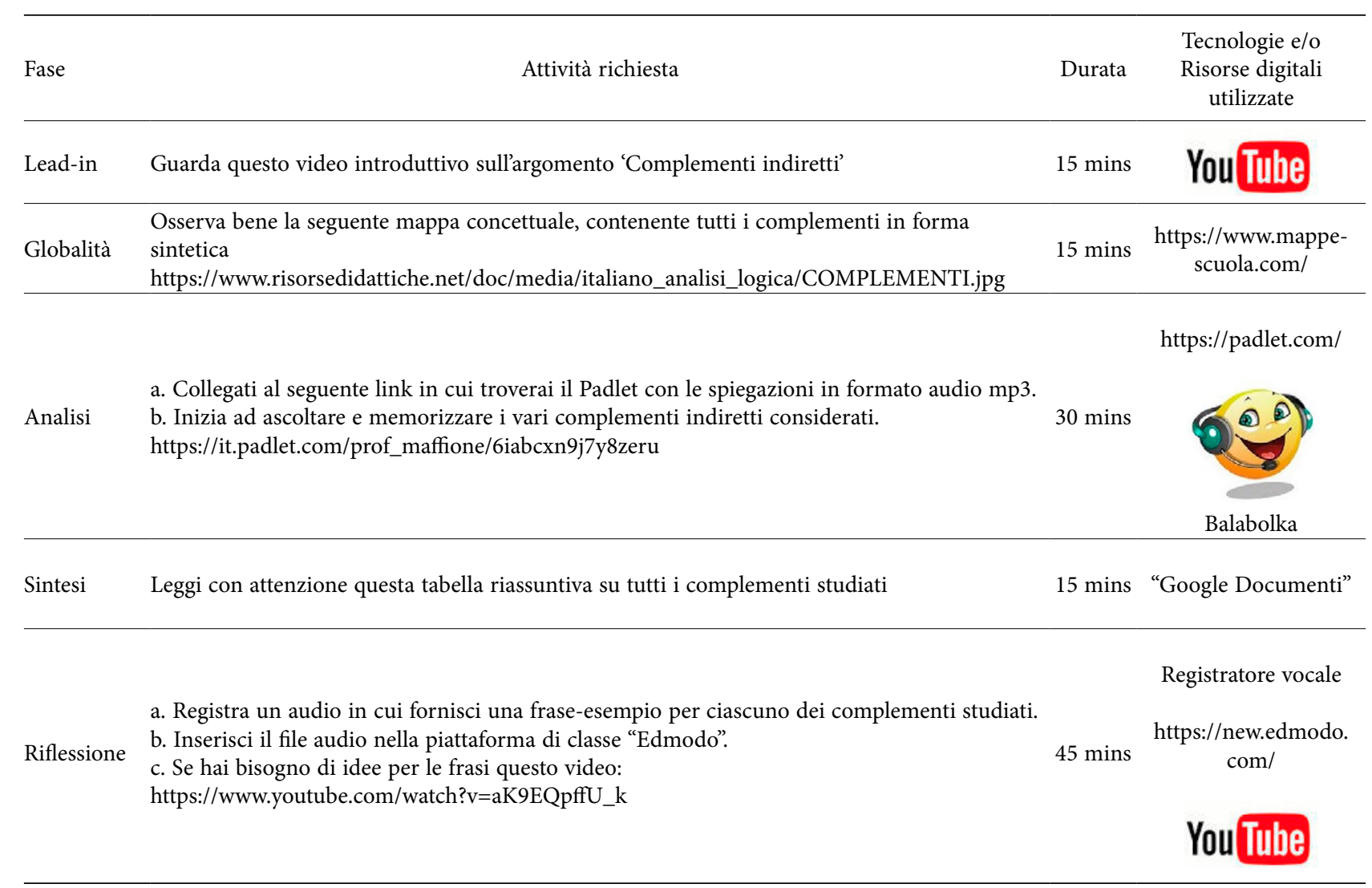

gie digitali, opportunamente contestualizzate e valorizzate hanno agito come efficaci facilitatori, rendendo 'digitale' il supporto didattico del docente specializzato, e permettendo così al processo di l'inclusione di non fermarsi.

\section{BIBLIOGRAFIA}

Balboni, E. P. (2002). Le sfide di Babele. Utet

Bezemer, J. \& Kress, G. (2016). Multimodality, Learning and Communication A social semiotic frame. Routledge.

Booth T. \& Ainscow M. (2002). Index for inclusion: Developing Learning and Participations in Schools. http://www. csie.org.uk/resources/translations/IndexEnglish.pdf

D’Alonzo, L. (2019) (a cura di). Dizionario di pedagogia speciale. Ed. Morcelliana.

Elliot B. (2008). E-pedagogy: Does e-learning require a new approach to teaching and learning? http://d. scribd.com/docs/22rc8wz72z067xrb1fpk.pdf

Gardner, H. \& Davis, K. (2014). Generazione App. La testa dei giovani e il nuovo mondo digitale. Feltrinelli.

Gordon, T. (1970/2014). Genitori Efficaci. La Meridiana.
Heidrun, D. (2019). Didattica Inclusiva. In D’Alonzo L. (2019) (a cura di), Dizionario di pedagogia speciale. Ed. Morcelliana, pp. 109-114.

Ianes, D. (2019). ICF- Classificazione internazionale del funzionamento, della disabilità e della salute. In D’Alonzo L. (2019) (a cura di), Dizionario di pedagogia speciale. Ed. Morcelliana, pp. 254-259.

McLoughlin, C. \& LeeJ, W. (a cura di) (2011). Web 2.0-based e-learning: Applying social informatics for tertiary teaching. IGI Global.

Rappoport, S., Rodríguez Tablado, M. S., Bressanello, M. (2020). Enseñar en tiempos de COVID-19. Una guía teórico-práctica para docentes, UNESCO.

\section{SITOGRAFIA}

https://www.mappe-scuola.com/

https://www.reteclassificazioni.it/portal_main.php?portal_ view=public_custom_page\&id $=25$

https://es.unesco.org/news/aprender-tiempos-covid-19 https://balabolka.it.softonic.com/ https://it.padlet.com/prof_maffione/6iabcxn9j7y8zeru 
https://www.risorsedidattiche.net/doc/media/italiano_ analisi_logica/COMPLEMENTI.jpg

https://www.youtube.com/watch?v=aK9EQpffU_k

https://www.reteclassificazioni.it/portal_main.php?portal_ view=public_custom_page\&id $=85$

https://repubblicadigitale.innovazione.gov.it/it/il-manifesto/

https://repubblicadigitale.innovazione.gov.it/it/il-programma/

https://www.youtube.com/watch?v=65YNG20zxiI

https://www.teamviewer.com/it/download/windows/

https://prezi.com/eafcwlgwqrya/copy-of-copy-of-learnprezi-fast $/$ ?present $=1$

https://www.youtube.com/watch?v=1MHevl-XBfQ

https://www.raiplayradio.it/programmi/adaltavoce/archivio/audiolibri/ 